\title{
Energy Distribution between Distance and Near Images in Apodized Diffractive Multifocal Intraocular Lenses
}

\author{
Fidel Vega, Francisco Alba-Bueno, and María S. Millán
}

\begin{abstract}
Purpose. To determine the energy distribution between the distance and near images formed in a model eye by spherical and aspheric apodized diffractive multifocal intraocular lenses (IOLs).

Methods. The IOL was inserted in a model eye with an artificial cornea with positive spherical aberration (SA) similar to that of the human cornea. The energy of the distance and near images, as a function of the pupil size, was experimentally obtained by image analysis. The level of SA on the IOL, which is pupil-sizedependent, was determined from simulations. The influence of the SA was deduced from results obtained in monofocal IOLs and by comparison of the experimentally obtained energy efficiency to theoretical results based solely on the diffractive profile of the IOL.
\end{abstract}

REsulTs. In contrast with theoretical predictions, the energy efficiency of the distance image strongly decreased for large pupils, because of the high level of SA in the IOL. The decrease was smaller in the apodized diffractive multifocal lens with aspheric design. As for the near image, since the diffractive zone responsible for the formation of this image was the same in the spherical and aspheric lenses and the apertures involved were small (and so the level of SA), the results turned out to be similar for both designs.

Conclusions. For large pupils, the energy efficiency of the distance image is strongly affected by the level of SA, although aspheric IOLs perform slightly better than their counterparts with a spherical design. For small pupils, there are no differences between the spherical and aspheric IOLs. (Invest Ophthalmol Vis Sci. 2011;52:5695-5701) DOI:10.1167/iovs.10-7123

$\mathrm{T}$ oday, the implantation of diffractive multifocal intraocular lenses (DMIOLs) to replace the crystalline lens has become a common procedure in cataract surgery. ${ }^{1}$ The principle of operation of most diffractive IOLs is to use the base lens curvature and the zero $(m=0)$ and first $(m=1)$ diffraction orders to achieve two focal points, sometimes referred to as optical powers, simultaneously along the optical axis. ${ }^{2,3}$ The power corresponding to the 0-order diffraction is used to image distant objects, and the other is used for near vision. This approach to providing the pseudophakic eye with the ability to see at different distances has an inherent drawback. The focused retinal image, provided by one of the lens powers, is

From the Department d'Òptica i Optometria, Universitat Politècnica de Catalunya, Terrassa, Spain.

Supported by project DPI2009-08879 from the Spanish Ministerio de Ciencia e Innovación y Fondos FEDER.

Submitted for publication December 22, 2010; revised May 6, 2011; accepted May 18, 2011.

Disclosure: F. Vega, None; F. Alba-Bueno, None; M.S. Millán, None

Corresponding author: Fidel Vega, Department d'Òptica i Optometria, Universitat Politècnica de Catalunya, Violinista Vellsola 37, 08222 Terrassa, Spain; fvega@oo.upc.edu. always overlaid by an out-of-focus image from the second lens power. This side effect may be visually disturbing, depending on the distance along the optical axis between the two images and their relative energy distribution. As a consequence, the contrast sensitivity in eyes implanted with a DMIOL may be worse than in eyes implanted with a monofocal IOL, ${ }^{4}$ and unwanted optical effects such as glare and/or halos are often reported by patients with DMIOLs in mesopic and escotopic conditions. ${ }^{5}$ For these reasons, it is interesting to characterize, both theoretically ${ }^{6}$ and experimentally, the optical performance of these IOLs in terms of the energy distribution between the distance and near images and its variation with the pupil diameter. This approach ${ }^{7}$ has rarely been used, in comparison with more common metrics such as the modulation transfer function (MTF) or the point spread function (PSF). ${ }^{8,9}$

The determination of the energy distribution between the two images is of particular relevance in the case of some DMIOLs, such as the apodized diffractive multifocal (ADM) AcrySof ReSTOR (Alcon Ltd., Fort Worth, TX), which was specifically designed with a twofold purpose: to reduce the glare and halo phenomena and to have an increasing distancedominant behavior for large pupil sizes by changing the energy balance between the distance and near images as a function of the eye pupil. In addition, some of these IOLs have an aspheric design, and there is great interest in determining, either at the testing bench ${ }^{10,11}$ or in clinical studies, ${ }^{12,13}$ the conditions and the degree to which the aspheric design might be advantageous versus a conventional spherical IOL, particularly when some studies have shown little or no benefit of aspheric IOLs in patients with small pupils. ${ }^{14,15}$

The goal of this study was to determine the energy distribution between distance and near images in ADMIOLs of spherical and aspheric design. We first calculated the energy distribution theoretically, paying special attention to the influence that the apodized diffractive profile of the IOL has on the energy efficiency of the distance and near diffraction orders. These results were compared with the energy distribution reported in research works supported by the manufacturer. ${ }^{16,17}$ Next, the energy distribution was experimentally determined from the analysis of the near and distance images obtained with the IOL inserted in a model eye that agrees with the one proposed by the International Organization for Standardization (ISO), ${ }^{18}$ except for the artificial cornea. Instead of the aberration-free cornea proposed in the ISO standard, our model eye has an artificial cornea that provides a realistic level of SA on the $\mathrm{IOL}^{19}$ that is similar to the values found in human corneas. ${ }^{20,21}$ The differences between the theoretical and experimental results allow us to determine the influence of the level of SA on the energy balance between distance and near images, particularly in eyes with large pupils. In addition, they permit us to establish the ocular conditions for which the aspheric design may be advantageous versus the spherical one. 


\section{MeTHods}

\section{Theoretical Energy Balance}

Although we will consider the AcrySof ReSTOR IOL (Alcon Ltd.) to be the reference lens in this work, the method outlined herein can be applied to any DMIOL. The AcrySof ReSTOR has an optical zone of 6-mm diameter with a hybrid diffractive-refractive design on its anterior surface (Fig. 1). The diffractive region covers the central $3.6 \mathrm{~mm}$ of the lens and is formed by 12 zones (a central disc and 11 concentric diffractive rings) that divert light simultaneously into distance and near powers. The outer region of the lens to the 6-mm edge is purely refractive and sends light to the distance power exclusively. The anterior IOL surface may be either spherical or aspheric, the latter introducing negative SA to compensate for the natural positive SA of human corneas. ${ }^{20,21}$ This compensation, expressed in terms of the $\mathrm{c}[4,0]$ Zernike coefficient, is as much as $0.20 \mu \mathrm{m}$ for a $6-\mathrm{mm}$ pupil. ${ }^{22}$

The relationship between the radii of the diffractive zones, measured from the optical axis $(r=0)$ and the add power, is given by the equation ${ }^{23}$.

$$
r_{i}^{2}=(2 i-1) \cdot \lambda \cdot \frac{1000}{D_{\text {add }}}
$$

where $i$ is the zone number ( $i=1-12), \lambda$ is the design wavelength, and $D_{\text {add }}$ is the add power (in diopters). For $\lambda$ of $550 \mathrm{~nm}$ and $D_{\text {add }}$ of $+4 \mathrm{D}$, the radius of the central disc $\left(r_{1}\right)$ is $0.375 \mathrm{~mm}$, whereas the outer diffractive ring $(i=12)$ has a radius $\left(r_{12}\right)$ of $1.8 \mathrm{~mm}$ (or $3.6 \mathrm{~mm}$ of diameter as stated above).

The most distinct characteristic of the design of the Acrysof ReSTOR IOL is that the height $\left(\boldsymbol{b}_{i}\right)$ of the diffractive steps at the zone boundaries gradually decreases from the center to the periphery, a design described as apodized by the manufacturer (Fig. 1). An apodization factor is given by Lee et al. ${ }^{23}$ :

$$
f_{i}^{\text {apodized }}=1-\left(\frac{r_{i}}{r_{12}}\right)^{3} \text { for } r_{i}=0, r_{1}, r_{2}, \ldots, r_{12}
$$

for which the step height reduction is given by

$$
\boldsymbol{h}_{i}=\boldsymbol{f}_{i}^{\text {apodized }} \cdot \boldsymbol{h}_{o}
$$

where $b_{\mathrm{o}}$ is the maximum height at the optical axis $(r=0)$. By setting $b_{\mathrm{o}}$ at $1.3 \mu \mathrm{m},{ }^{17}$ equation 3 produces a continuous reduction of the step height up to approximately $b_{11}=0.2 \mu \mathrm{m}$ and $b_{12}=0$ at the outer ring, in good agreement with the reported features of this type of IOL. ${ }^{16,17}$

The diffractive profile of the lens acts as an optical interface between the IOL material (refractive index $n_{\mathrm{IOL}}=1.55$ ) and the aqueous medium (refractive index $n_{\text {aqueous }}=1.336$ ), in which the lens is immersed. Because of the refractive index difference $n_{\mathrm{IOL}}-n_{\text {aqueous }}$ the light waves passing through different parts of the diffractive profile are phase shifted by different amounts. The induced phase shift $(\Phi)$ is proportional to the diffractive profile height $(b)$ :

$$
\Phi=\frac{2 \pi}{\lambda}\left(n_{\mathrm{IOL}}-n_{\text {aqueous }}\right) b
$$

Equation 4 is commonly expressed as a phase shift in wavelengths units as

$$
\alpha=\left(n_{\mathrm{IOL}}-n_{\text {aqueous }}\right) b / \lambda
$$

The maximum phase shift $\left(a_{i}\right)$ in a diffractive ring is

$$
\alpha_{i}=\left(n_{\mathrm{IOL}}-n_{\text {aqueous }}\right) \boldsymbol{b}_{i} / \lambda
$$

with $h_{i}$ calculated by equation 3 . The apodization of the diffractive profile of the DMIOLs implies that the value of $\alpha_{i}$ progressively decreases from $\alpha_{0}=0.51$ (i.e., nearly half wave) at the first diffractive zone to $\alpha_{i}=0.06$ at the outer diffractive ring. This fact has important implications on how the light is distributed between the $m=0$ (distance power) and $m=1$ (near power) diffraction orders as a function of the pupil aperture or equivalently as a function of the number of diffractive rings that are illuminated and take part in the distribution of the light between the $m=0$ and $m=1$ orders.

If $\alpha_{i}$ were constant for all the rings, the throughput efficiency (TE) of the $m=0$ and $m=1$ diffraction orders would be given by ${ }^{3,24}$ :

$$
T E_{m=0.1}=\sin c^{2}\left(m-\alpha_{\mathrm{i}}\right)
$$

However, since the value of $\alpha_{i}$ varies with the radius, the $T E$ for each $\alpha_{i}\left(T E_{m-0.1}^{i}\right)$ has to be weighted by a factor that corresponds to the $i$ th diffractive ring area. Therefore, the energy that the diffractive part of the IOL would divert from an incident plane wave into the $m=0$ and $m=1$ diffraction orders is calculated by means of linear combinations of the weighted contributions of all the rings:

$$
I_{m=o}^{\mathrm{diffractive}}=c_{\mathrm{te}} \cdot \sum_{i=1}^{12} A^{i} \cdot T E_{m=0}^{i}
$$

and

$$
I_{m=1}^{\mathrm{diffractive}}=c_{\mathrm{te}} \cdot \sum_{i=1}^{12} A^{i} \cdot T E_{m=1}^{i}
$$

where $c_{\mathrm{te}}$ is a proportionality constant, and $A^{i}$ is the area of the $i$ th diffractive ring. It is worth emphasizing that, when the IOL operates only with the first diffractive zone (i.e., $\alpha_{i}=\alpha_{0}$ ), there is a nearly equal a

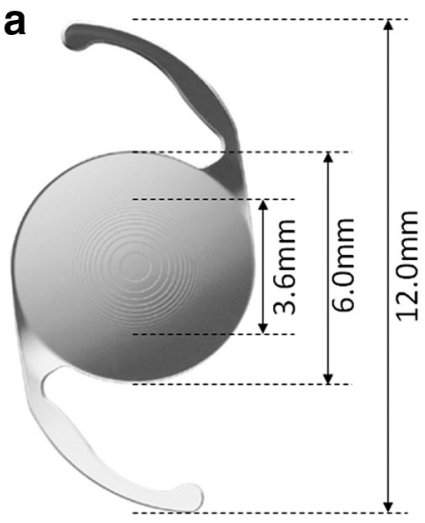

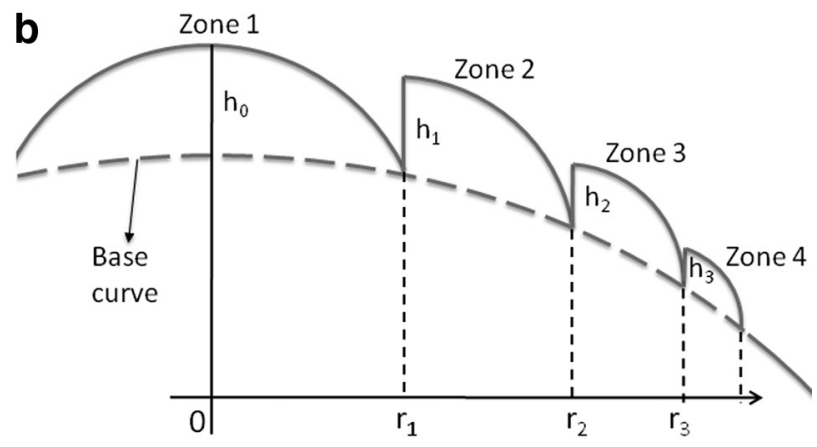

Figure 1. (a) The ADMIOL AcrySof ReSTOR (Alcon Ltd., Fort Worth, TX). (b) The first diffractive steps showing zone boundaries (dotted line) and the apodization process based on the variation of the step heights (solid line). 


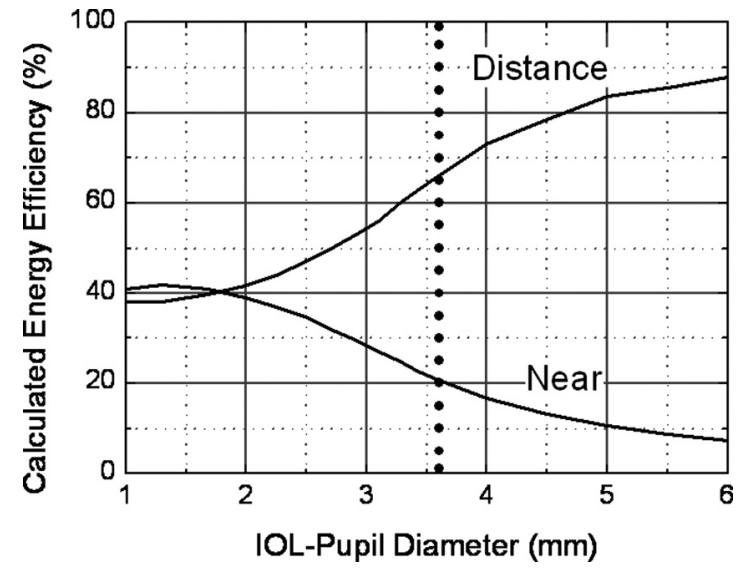

Figure 2. Theoretical energy efficiency of the distance and near powers of an ADMIOL. Dotted line: the diameter of the diffractive zone of the IOL $(3.6 \mathrm{~mm})$.

diffraction throughput efficiency for the distance $\left(T E_{m=0}^{0}=0.38\right)$ and near $\left(T E_{m=1}^{0}=0.43\right)$ powers. The progressive reduction of the phase shift of the waves $\alpha_{i}$ as they pass through the outer diffractive rings implies that $T E_{m=0}^{i}>T E_{m=1}^{i}$, and according to equations 8 and 9 , the energy sent to the distance power ( $m=0$ order) is favored at the expense of the near power ( $m=1$ order).

In the case of the purely refractive region of the ADMIOL, the light goes exclusively to the distance power (i.e., $T E_{m=0}^{\text {refractive }}=1$ ), and therefore the energy is simply:

$$
I_{m=0}^{\text {refractive }}=c_{\mathrm{te}} \cdot A^{\text {refractive }} \cdot T E_{m=0}^{\text {refractive }}
$$

where $A^{\text {refractive }}$ is the area of the illuminated refractive region of the ADMIOL. Then, in the case where the size $A_{\mathrm{IOL}}$ of the illuminated region of the IOL (referred to hereinafter as IOL-pupil diameter) would encompass the whole diffractive zone plus a fraction of the refractive one (i.e., $A_{\mathrm{IOL}}=\sum_{i=1}^{12} A^{i}+A^{\text {refractive }}$ ), the amounts of energy sent to either the distance and near powers would be, respectively:

$$
I_{m=o}=I_{m=o}^{\text {diffractive }}+I_{m=o}^{\text {refractive }}
$$

and

$$
I_{m=1}=I_{m=1}^{\text {diffractive }}
$$

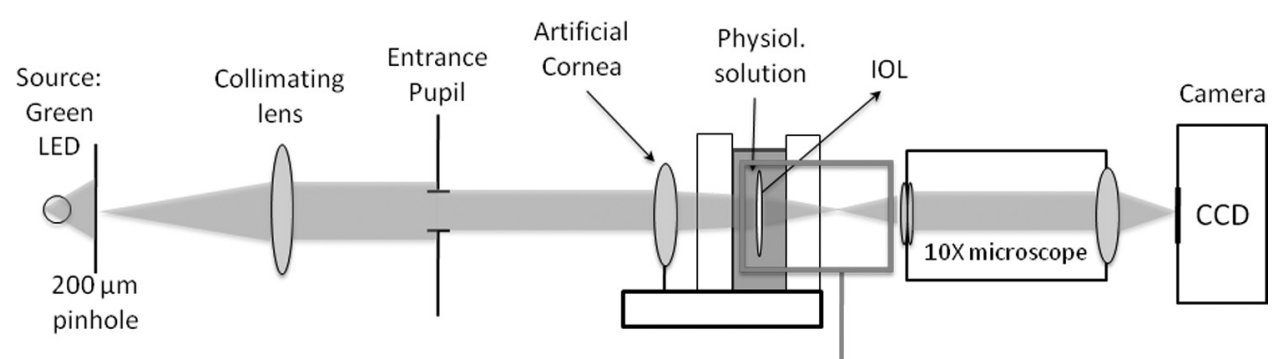

Figure 3. Setup used to obtain the distance and near images of a pinhole object. The detail shows how the ADMIOL works: The diffractive part of the lens (gray) sends light to both foci, whereas the outer purely refractive part sends light to the distance focus exclusively. which can be expressed in terms of energy efficiency as

$$
\frac{I_{m=o}}{I_{\mathrm{IOL}}^{\text {total }}}
$$

and

$$
\frac{I_{m=1}}{I_{\mathrm{IOL}}^{\text {total }}}
$$

where $I_{\mathrm{IOL}}^{\text {total }}$ is the total energy transmitted through the IOL-pupil. This energy is proportional to the IOL-pupil area $A_{\mathrm{IOL}}$, if any loss of energy caused by scattering in the diffractive steps ${ }^{25}$ is neglected:

$$
I_{\mathrm{IOL}}^{\mathrm{total}}=c_{\mathrm{te}} \cdot A_{\mathrm{IOL}}
$$

Incidentally, equation 15 implies a quadratic dependence of $I_{\mathrm{IOL}}^{\text {total }}$ with the IOL-pupil diameter, a well known fact in photography where the image illumination scales quadratically with the diaphragm diameter. ${ }^{26}$

We calculated the energy efficiencies according to equations 13 and 14, as a function of the IOL-pupil diameter of the AcrySof ReSTOR lens. The results, plotted in Figure 2, are in excellent agreement with those reported elsewhere ${ }^{16,17}$ and show that for small IOL-pupils, the energy was nearly equally divided between distance and near powers but that there was a gradual change in the energy balance to favor the distance power for large pupils. However, it must be emphasized that these calculations predict only the amount of the energy sent to either the distance or near powers for a particular IOL pupil, but they do not ensure that this energy would be properly focused on the respective images, the latter depending, among other factors, on the design of the base lens (aspheric versus spherical) from which the diffractive profile is made. This fact is especially relevant when the apodized diffractive IOL is inserted in any type of model eye with an aberrated cornea, ${ }^{27}$ the latter meaning that there would be a converging beam impinging on the IOL with a value of SA that depends on the size of the pupil aperture. This scenario may compromise the theoretical distance-dominant behavior of the AcrySof ReSTOR IOL for large pupils, particularly when dealing with IOLs of spherical design that tend to increase the optical aberrations of the eye. ${ }^{28}$

\section{Experimental Setup}

The energy distribution between the distance and near images can be obtained through image analysis using the setup shown in Figure 3. The quasi-monochromatic LED source (narrowband emission centered at $\lambda=521 \mathrm{~nm}$ ) with the $200 \mu \mathrm{m}$ pinhole object and the collimating 
TABle 1. Characteristics of the Model Eye

\begin{tabular}{lccc}
\hline \multicolumn{1}{c}{ Surface } & $\begin{array}{c}\text { Radius } \\
(\mathbf{m m})\end{array}$ & $\begin{array}{c}\text { Thickness } \\
(\mathbf{m m})\end{array}$ & $\begin{array}{c}\text { Refractive } \\
\text { Index }\end{array}$ \\
\hline Object & & Infinity & 1 \\
Cornea front & 35.99 & 4.29 & 1.4599 \\
Cornea back & -35.99 & 8.6 & 1 \\
Window front & Flat & 6 & 1.5185 \\
Window back & Flat & 6.25 & 1.336 \\
Iris pupi $^{*}$ & Flat & 10 & 1.336 \\
Window front & Flat & 6 & 1.5185 \\
Window back & Flat & 9.24 & 1 \\
Image & - & - & - \\
\hline
\end{tabular}

* Position where the IOL is inserted.

lens produce a collimated beam to illuminate the model eye (formed by the iris diaphragm, the artificial cornea, and the wet cell) where the IOL is inserted. The features of the model eye are given in Table 1 . The IOL's holder is mounted in high-precision linear ( $x, y$, and $z$ axis) and rotation (tilt and tip) stages. The pinhole object is imaged by the model eye with the ADMIOL in two planes separated along the optical axis. A $10 \times$ infinite corrected microscope mounted in a translation holder is used to select either the distance or the near image and magnify it onto an 8-bit CCD camera. Before any measurement, the IOL is centered without any tilt/tip with the aid of a $6 \times$ objective lens coupled to a CCD video camera. To this end, the iris diaphragm is closed as much as possible and the light scattered onto the IOL surface is used to ensure the proper position of the lens (Fig. 4). Further details of the experimental setup can be found elsewhere. ${ }^{29}$

The iris diaphragm, which has a variable aperture, is placed in front of the artificial cornea as the entrance pupil (EP), to control the size of the beam on the artificial cornea and thus the level of SA introduced by the model eye (without the IOL). In addition, when the IOL is inserted, the EP diameter also determines the size of the beam on the IOL (the IOL-pupil). A linear relationship is obtained ${ }^{29}$ between the diameters $\Psi$ of the EP and the IOL-pupil:

$$
\Psi_{\mathrm{IOL}-\text { pupil }}=0.52 \Psi_{\mathrm{EP}}
$$

We used commercial optical design software (Zemax Development Corporation, San Diego, CA) to obtain the dependence of the SA of the wavefront that leaves the artificial cornea and impinges on the IOL as a function of the EP diameter. The results, expressed in terms of the values of the Zernike c[4,0] coefficient and plotted in Figure 5, show

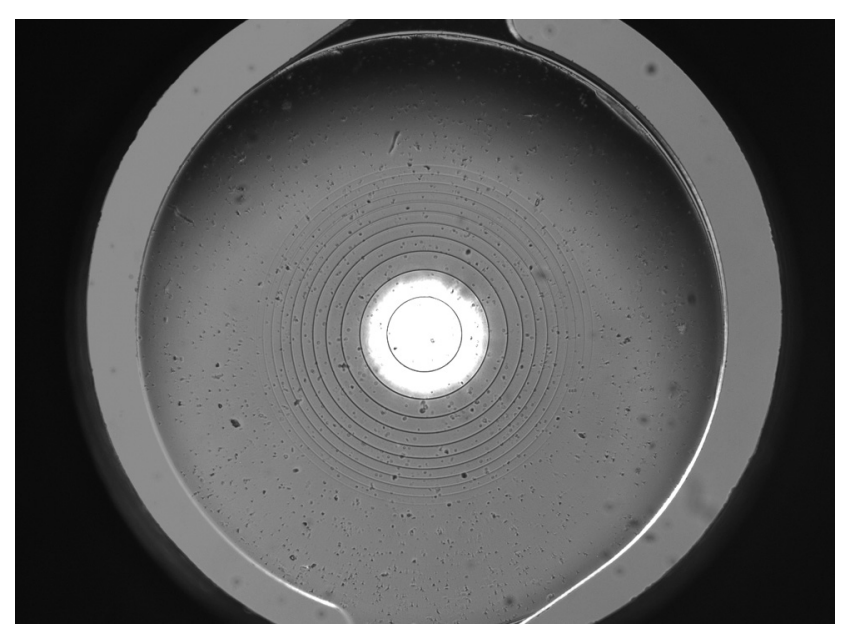

FIGURE 4. Image showing the centering of an ADMIOL. The image is obtained with a $6 \times$ objective lens. The bright spot corresponds to the beam coming from the artificial cornea.

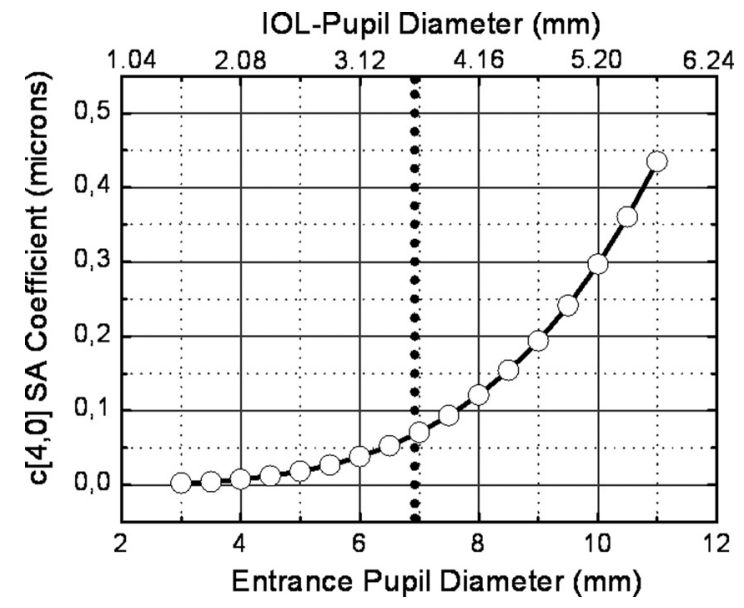

FIGURE 5. Calculated value of the Zernike c[4,0] SA coefficient as a function of the EP diameter. Top axis: beam size at the IOL position or IOL-pupil diameter, calculated by equation 16. Dotted line: the diameter of the diffractive zone of the ADMIOL $(3.6 \mathrm{~mm})$.

that for EP diameters up to $7 \mathrm{~mm}$, which actually means an IOL-pupil diameter of $\sim 3.6 \mathrm{~mm}$ (i.e., the entire apodized diffractive zone), the SA contribution was relatively small. For larger pupils, there was a significant increase in the level of SA. Thus, for EP diameters above $9 \mathrm{~mm}$ (or IOL-pupil diameter $>4.7 \mathrm{~mm}$ ) the value of $c[4,0]$ was larger than 0.2 $\mu \mathrm{m}$, which corresponds to the maximum value of SA for which the aspheric Acrysof IOL design is theoretically able to compensate. ${ }^{27}$ From now on, references to small or large IOL-pupil diameters will be understood to indicate that the diameters are smaller or larger than 3.6 $\mathrm{mm}$, respectively.

Examples of the near and distance images of the pinhole object obtained with an AcrySof RESTOR ADMIOL are shown in Figures 6a and $6 \mathrm{~b}$, respectively. Both images consist of the focused image of the pinhole (labeled $I_{\text {pinh }}$ in Fig. 6) surrounded by a more or less defined blurred halo (labeled $I_{\text {backg }}$ in Fig 6). This background corresponds primarily to the overlaying defocused image but, in the case of the distance image (Fig. 6b), it will be shown that there is an additional contribution that strongly depends on the level of SA on the IOL.

The energy of the image in the focused pinhole region $\left(I_{\text {pinh }}\right)$ only and the energy of the total image that comprises the pinhole plus background regions $\left(I_{\text {total }}=I_{\text {pinh }}+I_{\text {backg }}\right)$ are obtained by integration of the pixel gray level in the corresponding regions:

$$
I_{R}=\sum_{n \text { pixel }}^{n \in R} g(n)
$$

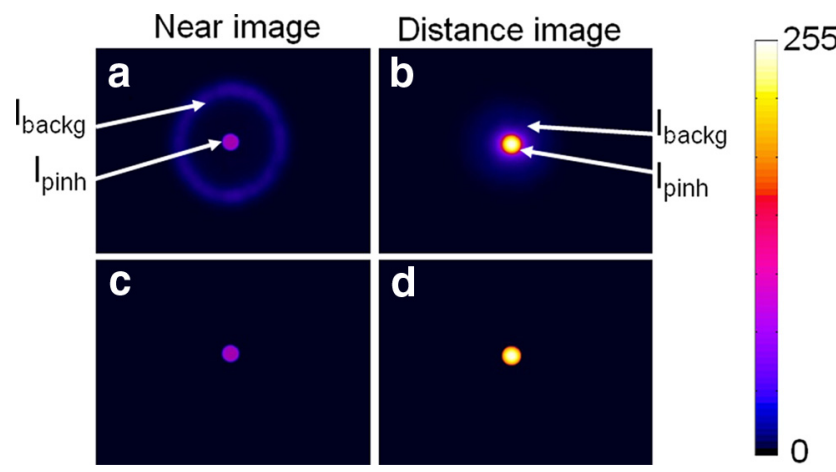

FigURE 6. (a, b) Near and distance images experimentally obtained with an ADMIOL in the model eye. Arrows: the pinhole (pinh) and background (backg) regions, respectively. Pseudocolor is used for a better visualization of the contrast in the background. (c, d) Same as (a, b) after removing the background. 
where $R$ is either the pinhole region or the total image $(R=$ pinh, total), $n$ is a pixel contained in the $R$ region, and $g(n)$ is the pixel gray level. Since the images are blurred because of the background, determining the borders of the region that correspond only to the focused pinhole is not a straightforward matter. An edge-detection algorithm was used to unambiguously define a region of interest (ROI) and remove all the background contribution outside this ROI (Figs. 6c, 6d). Then, the $I_{\mathrm{pinh}}$ is calculated from these filtered images, according to equation 17 .

Two types of multifocal IOLs were used in this study: the AcrySof ReSTOR SN60D3 with an anterior spherical apodized diffractive surface and the AcrySof ReSTOR SN6AD3 (both from Alcon Ltd.), which shares with the SN60D3 the same apodized diffractive design but with an aspheric surface. The manufacturer provides lenses with base optical power from +10.0 through +30.0 in 0.5 -D increments. The add power for near vision is in all cases $4.0 \mathrm{D}$. In our study, all the analyzed lenses had the same base optical power for the distance focus: $20.0 \mathrm{D}$. The counterpart spherical and aspheric monofocal IOLs (SN60AT and SN60WF, respectively) of $20.0 \mathrm{D}$ were also studied for the sake of comparison. At least two lenses of each type were tested, to guarantee the repeatability of the measurements.

\section{Results}

The experimental procedure was applied to monofocal and multifocal IOLs, although it must be pointed out that, in the case of the monofocal IOLs, there is only one focus, and thus no other defocused image can contribute to the background.

The variation of the energies $I_{\text {total }}$ and $I_{\text {pinh }}$ obtained with the monofocal spherical (SN60AT) and aspheric (SN60WF) IOLs is plotted, as a function of the EP diameter, in Figure 7. The corresponding image energy efficiencies, defined as $I_{\text {pinh }}$ $I_{\text {total }}$, are plotted in Figure 8. To make the comparison of results easier, $I_{\text {total }}$ and $I_{\text {pinh }}$ are normalized to the value of $I_{\text {total }}$ obtained with the largest EP diameter $(11 \mathrm{~mm})$. The results in Figure 7 show a quadratic dependence of $I_{\text {total }}$ with the EP diameter, in good agreement with the theoretical values predicted by equation 15 . More interesting, for both types of IOLs and small EP diameters up to $6 \mathrm{~mm}$ (at which the level of SA on the IOL is small), the energy $I_{\text {pinh }}$ was nearly the same as the $I_{\text {total }}$, which means that the background contribution was negligible in these conditions, no matter the type (spherical or aspheric) of monofocal IOL. As a consequence, high image

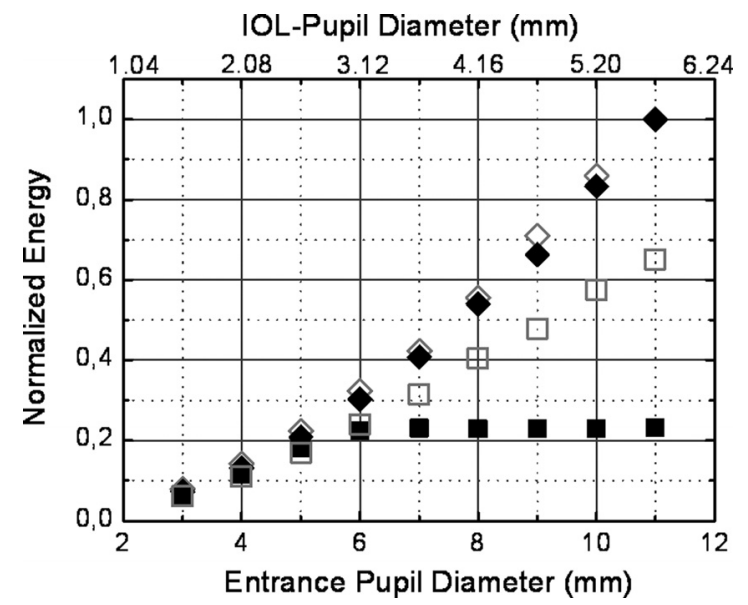

FIGURE 7. Energies $I_{\text {total }}(\diamond, \diamond)$ and $I_{\text {pinh }}(\square, \square)$ measured in the image plane of monofocal IOLs as a function of the EP pupil diameter. Filled symbols: results obtained with the spherical SN60AT IOL; open symbols: results obtained with the aspheric SN60WF IOL. Top axis calculated as in Figure 5.

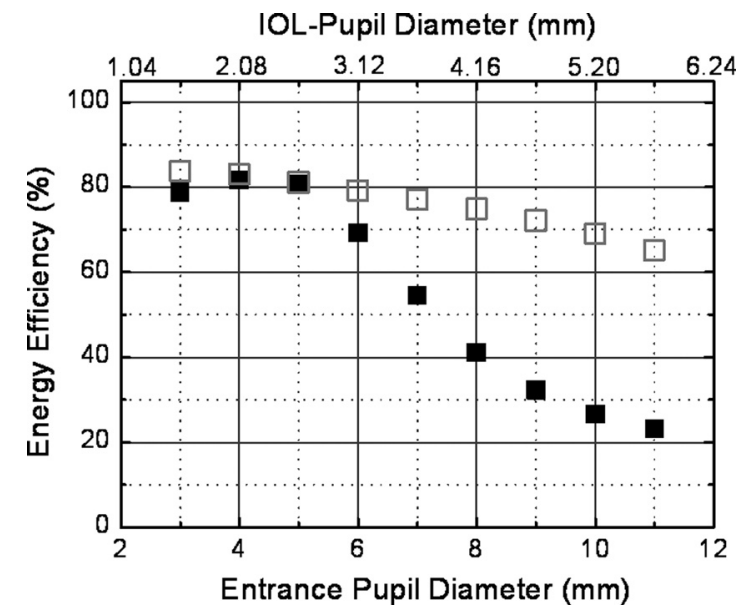

FIGURE 8. Energy efficiency $\left(I_{\text {pinh }} / I_{\text {total }}\right)$ as a function of the EP diam-

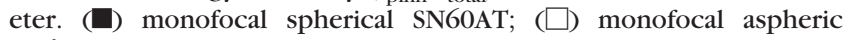
SN60WF. Top axis: calculated as in Figure 5.

efficiencies on the order of $80 \%$ were obtained with small apertures for both IOLs, as is shown in Figure 8.

At larger EP diameters, there was quite different behavior between spherical and aspheric IOLs. In the case of the spherical SN60AT, the energy $I_{\text {pinh }}$ remained constant, even though the measured $I_{\text {total }}$ increased (Fig. 7). This result implies that, when the EP is opened, most of the additional available energy is not sent to the pinhole image but is wasted on the background, and consequently, a dramatic reduction in image efficiency occurs (see Fig. 8). For the aspheric SN60WF, the larger the EP the larger the value of $I_{\text {pinh }}$, but the increase occurred with a slope smaller than the increase in $I_{\text {total }}$ (Fig. 7), which implies a moderate reduction in its efficiency for larger EP diameters, as is shown in Figure 8.

The same type of measurements were performed for the spherical (SN60D3) and aspheric (SN6AD3) ADMIOLs. The results for the distance and near images are plotted in Figure 9, as a function of the EP diameter. As was found to be the case with the monofocal IOLs, the energy $I_{\text {total }}$ (in the case of both distance and near images) scaled quadratically with the EP diameter.

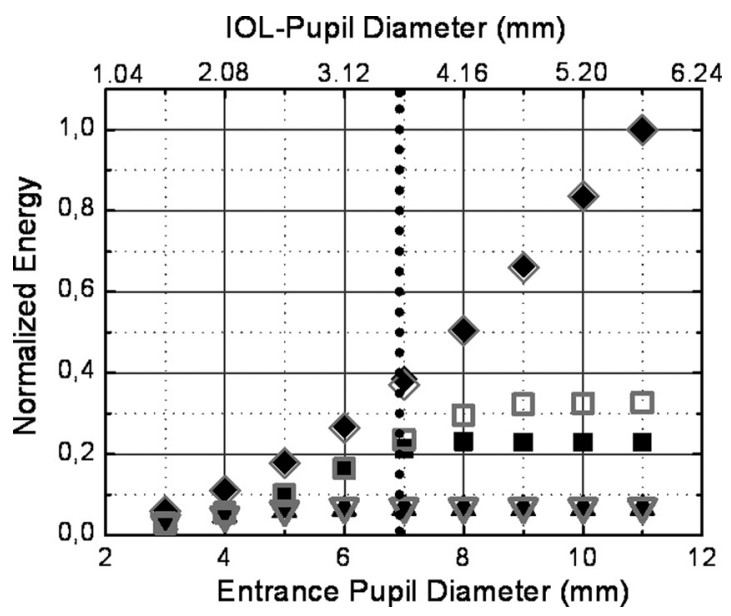

Figure 9. Energies $I_{\text {total }}(\diamond, \diamond)$ and $I_{\text {pinh }}$ measured in the near $(\boldsymbol{\Delta}, \nabla)$ and distance $(\square, \square$ ) image planes of an ADMIOL as a function of the EP pupil diameter. Filled symbols: results obtained with the spherical SN60D3; open symbols: results obtained with the aspheric SN6AD3. Dotted line: the diameter of the diffractive zone of the IOLs $(3.6 \mathrm{~mm})$ is indicated. Top axis: calculated as in Figure 5. 
More interesting, in the case of the near images, there was practically no difference between the energy $I_{\text {pinh }}$ measured in the spherical and aspheric multifocal IOLs. It increased slightly for EP diameters up to $6 \mathrm{~mm}$ (i.e., up to IOL-pupils of $\sim 3.6 \mathrm{~mm}$ that corresponds to the diffractive zone of the IOL) and then kept a low constant value for larger pupils. Since on the other hand the energy $I_{\text {total }}$ of the near image increased with the EP (see Fig. 9), the energy efficiency $I_{\text {pinh }} / I_{\text {total }}$ strongly decreased for large pupils, as is shown in Figure 10.

With distance images and large EP diameters, the design of the multifocal IOLs (spherical versus aspheric) produced some differences in the experimental results. For the spherical SN60D3, as is plotted in Figure 9, the value of $I_{\text {pinh }}$ first increased with the EP diameter (up to $7 \mathrm{~mm}$ ) and then kept a constant value for larger pupils. The results of the aspheric SN6AD3 IOL followed a similar tendency, but the constant value of $I_{\text {pinh }}$ measured for large pupils was slightly higher than the value measured for the spherical SN60D3 IOL.

Expressing these results in terms of energy efficiency (Fig. 10), we see that for distance images, both IOLs (spherical and aspheric) behaved very similarly and in agreement with theoretical calculations only up to moderate pupil diameters (IOLpupils of $\sim 3.6 \mathrm{~mm}$ for the spherical and $\sim 4.2 \mathrm{~mm}$ for the aspheric). In these conditions, which correspond to reduced levels of SA on the IOL, the maximum energy efficiency achieved was $\sim 60 \%$. For larger pupils when the contribution of the refractive part of the IOLs to the distance image was gaining in importance, there was a clear reduction in energy efficiency for both IOLs, in contrast with the theoretically predicted distance-dominant behavior of the Acrysof ReSTOR IOLs, also plotted for comparison in Figure 10.

\section{Discussion}

The results obtained with monofocal IOLs can be explained by taking into account both the level of the SA as a function of EP (Fig. 5) and the IOL design. Thus, for small EP diameters, when the effects of the SA were low, there were no relevant differences in the performance of the aspheric versus the spherical monofocal IOL. This seems to be in good agreement with clinical studies ${ }^{15}$ that did not find significant differences in contrast sensitivity between aspheric and spherical IOLs for small apertures.

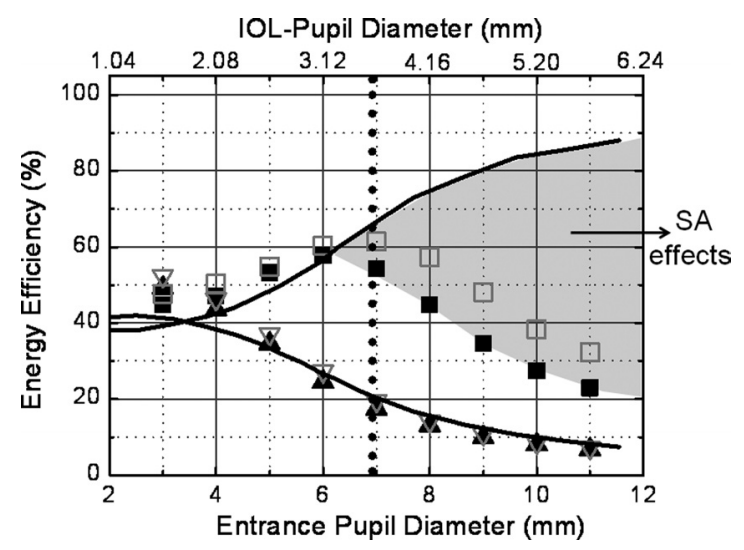

FIGURE 10. Energy efficiency $\left(I_{\text {pinh }} / I_{\text {total }}\right)$ of the near $(\boldsymbol{\Lambda}, \nabla)$ and distance $(\boldsymbol{\square}, \square)$ images as a function of the EP diameter of an ADMIOL Filled symbols: results obtained with the spherical SN60D3; open symbols: results obtained with the aspheric SN6AD3. Solid lines: theoretical calculations, shown in Figure 2, are included. Dotted line diameter of the diffractive zone of the IOLs $(3.6 \mathrm{~mm})$. Top axis: calculated as in Figure 5.
For larger pupils, however, there are higher levels of SA on the IOLs. The spherical IOL introduces additional positive SA that adds to the one produced by the artificial cornea, ${ }^{28}$ and, as a consequence, more and more energy goes to the background when the EP diameter increases, which strongly reduces its efficiency (Fig. 8). On the contrary, the aspheric IOL tends to partially counteract the SA of the artificial cornea and manages still to focus a good amount of the additional energy in the pinhole region of the image, although it cannot impede a certain reduction of its energy efficiency by large pupils. The best performance of the aspheric IOLs in terms of energy efficiency for large pupils would be in agreement with clinical findings ${ }^{13,30}$ that showed that patients with implanted aspheric IOLs have better contrast sensitivity in mesopic conditions (i.e., large pupil diameters) than do those with spherical IOLs.

In the case of the ADMIOLs and for the near image, there was not any significant difference between the results obtained with the aspheric and spherical IOLs (Figs. 9, 10). This was not unexpected, considering that the maximum aperture involved in the formation of the near image corresponds to the diffractive zone of the IOL, which is relatively small, and so is the level of SA on the IOL (Fig. 5). Once the diffractive zone of the lens is fully illuminated, there is no way to send more energy to the near image, and, as a consequence, $I_{\text {pinh }}$ in the near image remains constant (Fig. 9), independent of the EP diameter and the particular design (spherical or aspheric) of the IOL. In these conditions, the aspheric design of the SN6AD3 proves to be no advantage, in terms of near image energy efficiency, over the spherical design of the SN60D3. These results seem to be in agreement with those in clinical studies ${ }^{14,15}$ that have shown that for small pupils there is little or no benefit in using aspheric IOLs.

Additional confirmation of the small influence that the SA has in the near image efficiency is provided by the excellent agreement between the experimental and theoretical results shown in Figure 10, the latter calculated without including any SA effect.

As for the distance image and large pupils, there were significant differences between the experimental results and the theoretical ones (Fig. 10), which put into question the distance-dominant behavior of the IOL in the presence of SA. Our results show that most of the additional energy available for the distance image when the EP diameter increases does not end up in the pinhole image but in the background. This adverse effect is even worse in the case of the spherical multifocal IOL, which cannot properly counteract the SA produced by the model eye.

\section{Conclusions}

An experimental method based on the analysis of the energy of the distance and near images formed by either spherical or aspheric ADMIOLs in a model eye was developed to obtain the energy distribution between these images as a function of pupil diameter. Measurements of monofocal spherical and aspheric IOLs put into evidence the influence of the level of SA in the IOL efficiency. In the case of the ADMIOLs and for the distance image, the results show that, with large EP diameters, the level of SA on the IOLs is too high to correctly focus the available energy on the image. This effect occurs even for the multifocal IOL with aspheric design (SN6AD3) and thus, in contrast with the theoretical predictions, there is a strong reduction in energy efficiency for distance images and large pupils. In the case of the near image, similar results are obtained with both types of spherical and aspheric multifocal IOLs, most likely because they share the design of the diffractive part, and the apertures involved in the near image 
formation are always small enough to minimize the influence of the SA.

\section{References}

1. Kohnen T, Allen D, Boureau C, et al. European multicenter study of the AcrySof ReSTOR apodized diffractive intraocular lens. $O p h$ thalmology. 2006;113:578-584.

2. Larsson M, Beckman C, Nyström A, Hard S, Sjöstrand J. Optical properties of diffractive, bifocal, intraocular lenses. Appl Opt. 1992;31:2377-2384.

3. Cohen AL. Practical design of a bifocal hologram contact lens or intraocular lens. Appl Opt. 1992;31:3750-3754.

4. Schmidinger G, Simader C, Dejaco-Ruhswurm I, Skorpik C, Pieh S. Contrast sensitivity function in eyes with diffractive bifocal intraocular lenses. J Cataract Refract Surg. 2005;31:2076-2083.

5. Vingolo EM, Grenga P, Iacobelli L, Grenga R. Visual acuity and contrast sensitivity: AcrySof ReSTOR apodized diffractive versus AcrySof SA60AT monofocal intraocular lenses. J Cataract Refract Surg. 2007;33:1244-1247.

6. Castignoles F, Flury M, Lepine T. Comparison of the efficiency, MTF and chromatic properties of four diffractive bifocal intraocular lens designs. Opt Express. 2010;18:5245-5256.

7. Ravalico G, Parentin F, Sirotti P, Baccara F. Analysis of light energy distribution by multifocal intraocular lenses through an experimental optical model. J Cataract Refract Surg. 1998;24:647-652.

8. Eppig T, Scholz K, Lagenbucher A. Assesing the optical performance of multifocal (diffractive) intraocular lenses. Opbthalmic Pbysiol Opt. 2008;28:467-474.

9. Pieh S, Fiala W, Malz A, Stork W. In vitro Strehl ratios with spherical, aberration-free, average, and customized spherical aberration-correcting intraocular lenses. Invest Opbthalmol Vis Sci. 2009;50:1264-1270.

10. Pieh S, Marvan P, Lackner B, et al. Quantitative performance of bifocal and multifocal intraocular intraocular lenses in a model eye. Arch Opbthalmol. 2002;120:23-28.

11. Terwee T, Weeber H, Van der Mooren M, Piers P. Visualization of the retinal image in an eye model with spherical and aspheric, diffractive, and refractive multifocal IOLs. J Refract Surg. 2008;24: 223-232.

12. de Vries NE, Webers CAB, Verbakel F, et al. Visual outcome and patient satisfaction after multifocal intraocular lens implantation: aspheric versus spherical design. J Cataract Refract Surg. 2010; 36:1897-1904.

13. Kohnen T, Klaproth OK, Bühren J. Effect of intraocular lens asphericity on quality of vision after cataract removal: an intraindividual comparison. Opbthalmology. 2009;116:1697-1706.

14. Muñoz G, Albarrán-Diego C, Montés-Micó R, Rodriguez-Galietero A, Alió JL. Spherical aberration and contrast sensitivity after cata- ract surgery with the Tecnis Z9000 intraocular lens. J Cataract Refract Surg. 2006;32:1320-1327.

15. Kasper T, Bühren J, Kohnen T. Visual performance of aspheric and spherical intraocular lenses: intraindividual comparison of visual acuity, contrast sensitivity, and higher order aberrations. J Cataract Refract Surg. 2006;32:2022-2029.

16. Davison JA, Simpson MJ. How does the ReSTOR lens work? Rev Refract Surg. 2004;18-20.

17. Davison JA, Simpson MJ. History and development of the apodized diffractive intraocular lens. J Cataract Refract Surg. 2006;32:849858 .

18. International Organization for Standardization (ISO), ISO 11979-2 ophthalmic implants, intraocular lenses Part 2: optical properties and test methods (ISO 1999).

19. Vega F, Millán MS, Wells B. Spherical lens versus aspheric artificial cornea for intraocular lens testing. Optics Lett. 2010;35:15391541.

20. Guirao A, Redondo M, Artal P. Optical aberrations of the human cornea as a function of age. J Opt Soc Am A. 2000;17:1697-1702.

21. Wang L, Dai E, Koch DD, Nathoo A. Optical aberrations of the human anterior cornea. J Cataract Refract Surg. 2003;29:15141521 .

22. Schwiegerling J. Intraocular lenses. In: Bass M, Enoch JM, Lakshminarayanan V, eds. Vision and Vision Optics. 3rd ed. New York: 2010:21.1-21.27. Handbook of Optics; vol 3.

23. Lee C, Simpson MJ. Diffractive Multifocal Ophthalmic Lens. U.S. Patent 5,699,142. December 16, 1997.

24. Cohen AL. Diffractive bifocal lens designs. Optom Vis Sci. 1993; 70:461- 468 .

25. de Vries NE, Franssen L, Webers CAB, et al. Intraocular straylight after implantation of the multifocal AcrySof ReSTOR SA60D3 diffractive intraocular lens. J Cataract Refract Surg. 2008;34:957962.

26. Kingslake R. Optics in Pbotography. Bellingham, WA: SPIE Press; 1992.

27. Norrby S, Piers P, Campbell Ch, van der Mooren M. Model eyes for evaluation of intraocular lenses. Appl Opt. 2007;46:6595-6605.

28. Packer M, Fine H, Hoffman RS. Aspheric intraocular lens selection: the evolution of refractive cataract surgery. Curr Opin Opbthalmol. 2008; 19:1-4.

29. Alba-Bueno F, Vega F, Millán MS. Design of a test bench for intraocular lens optical characterization. J Phys Conf Ser. 2011; 274:012105-012111.

30. Tzelikis PF, Akaishi L, Trinidade FC, Boteon JE. Spherical aberration and contrast sensitivity in eyes implanted with aspheric and spherical intraocular lenses: A comparative study. Am J Ophthalmol. 2008; 145:827-833. 